\title{
A new genus, two new species and a new record of subfamily Cecidophyinae (Acari, Eriophyidae) from China
}

\author{
Guo-Quan Wang ${ }^{1, \dagger}$, Sui-Gai Wei ${ }^{1}$, , Ding Yang ${ }^{1, \S}$ \\ I Department of Plant Protection, Agricultural College, Guangxi University, Nanning, China \\ † urn:lsid:zoobank.org:author:2FAFA732-C8F7-4823-949A-EF81D6B6A2F4 \\ † urn:lsid:zoobank.org:author:54A504AB-53B4-4181-BFE1-675ECE541FFA \\ § urn:lsid:zoobank.org:author:FD9077E0-D8D5-4A3A-80FD-2862726AA066 \\ Corresponding author: Guo-Quan Wang (wgq1230@yahoo.com.cn)
}

Academic editor: A. Bochkov | Received 9 January 2012 | Accepted 27 March 2012 | Published 5 April 2012

urn:lsid:zoobank.org:pub:D7CCD2A1-5564-4190-A935-3E70BOBE0E20

Citation: Wang G-Q, Wei S-G, Yang D (2012) A new genus, two new species and a new record of subfamily Cecidophyinae (Acari, Eriophyidae) from China. ZooKeys 180: 9-18. doi: 10.3897/zookeys.180.2641

\begin{abstract}
A new genus and two new species belonging to subfamily Cecidophyinae, namely Kyllocarus reticulatus gen. n., sp. n. infesting Lithocarpus brevicaudatus (Skan) Hay. (Fagaceae) and Gammaphytoptus schimae sp. n. infesting Schima superba Gardn \& Champ. (Theaceae) are described and illustrated. Both new species are vagrants on their respective host plants. Cecidophyes digephyrus Keifer, 1966 is newly recorded for China.
\end{abstract}

\section{Keywords}

Eriophyoidea, eriophyoid mites, Cecidophyini, Colomerini, taxonomy, China

\section{Introduction}

The subfamily Cecidophyinae holds two tribes, Cecidophyini and Colomerini, which were differentiated by the former scapular tubercles and setae are absent and the later tubercles and setae are present. So far, sixteen genera and thirty-three species are known from China (Huang 2001; Huang and Cheng 2005; Huang and Wang 2004, 2009;

Copyright Guo-Quan Wang et al. This is an open access article distributed under the terms of the Creative Commons Attribution License 3.0 (CC-BY), which permits unrestricted use, distribution, and reproduction in any medium, provided the original author and source are credited. 
Kuang 1987, 1995, 1997, 1998; Kuang and Hong 1992, 1995; Kuang and Luo 1992; Kuang, Luo and Wang 2005; Li et al. 2009; Song et al. 2008; Wang et al. 2009; Wei et al. 2009; Xue et al. 2009). Herein, one new genus and new species Kyllocarus reticulatus gen. n., sp. n., in the tribe Cecidophyini and another new species, Gammaphytoptus schimae sp. n., in the tribe Colomerini are described and illustrated.

\section{Materials and methods}

Specimens were located with the aid of a magnifying glass on plant material in the field, and specimens were collected into and preserved in a sucrose-ethanol solution (75\%). The mites were cleared in Nesbitt's solution and mounted in Heinze medium on glass slides at room temperature according to Kuang (1986). Specimens were measured follows de Lillo et al. (2010). The morphological terminology and the generic classification follow Amrine et al. (2003).

Type specimens are deposited in the Department of Plant Protection, Guangxi University, Nanning. All measurement units are in micrometers $(\mu \mathrm{m})$ and rounded off to the nearest full number, and are lengths when not specified. Specimens were examined with an Olympus CX41 (Philippines) microscope with phase contrast. The number of measured specimens is given in parentheses.

\section{Taxonomy}

Tribe Cecidophyini Keifer, 1966

Genus Cecidophyes Nalepa, 1887

Type species. Phytopus galii Karpelles, 1884.

Cecidophyes digephyrus Keifer, 1966, rec. n.

http://species-id.net/wiki/Cecidophyes_digephyrus

Diagnosis. Body fusiform. Prodorsal shield with frontal lobe present; median, admedian lines and submedian lines complete, connected with three transverse lines forming network; scapular tubercles and setae absent. Coxisternal plates sculptured with lines. Legs with normal segments and usual setae, tarsal empodium entire 6-rayed, tarsal solenidion knobbed. Dorsal opisthosoma evenly rounded, dorsal annuli 62-68, with elongated microtubercles; ventral annuli 62-68, with rounded microtubercles, setae $h 1$ absent. Female genital near coxisternal plates, coverflap with two rows of ridges.

Material examination. 10 females, Qingliangfeng National Nature Reserve, Lin'an City $\left(30^{\circ} 10^{\prime} \mathrm{N}, 119^{\circ} 07^{\prime} \mathrm{E}\right)$, Zhejiang Province, China, 24. VII. 2007, from Quercus sp. (Fagaceae), collected by Guo-Quan Wang, mounted on individual slide. 
Distribution. USA, China (Zhejiang).

Relation to host. The mites are vagrants on the surfaces of the leaves, no visible damage seen.

Remarks. Up to date, nine species of Cecidophyes have been known infesting Quercus spp.: C. caliquerci (Keifer, 1944) infesting Q. lobata Nee and Q. marilandica Muen., C. digephyrus Keifer, 1966 infesting Q. vaccinifolia Kell., C. lyrata Keifer, 1959 infesting Q. lyrata Walt., C. pusilla Keifer, 1962 infesting Q. falcata Michx, C. quercialbae Keifer, 1959 infesting Q. alba L., C. querciphagus (Keifer, 1939) infesting Q. sp., C. reticulatus Livshitz, Mitrofanov et Vasil'yeva, 1979 infesting Q. pubescens Willd., C. tampae Keifer, 1966 infesting Q. virginiana and C. tristernalis (Nalepa, 1898) infesting Q. cerris L. Among them, only one species, C. tampae occurred in China. C. digephyrus is second Cecidophyes species from China (Keifer 1939, 1944, 1959a, b, 1962, 1966; Livshitz et al. 1979; Nalepa 1898; Xue et al. 2009).

\section{Genus Kyllocarus Wang, Wei \& Yang, 2012, gen. n.}

urn:lsid:zoobank.org:act:91681721-9DF7-483B-941C-C882DBF8A4FF http://species-id.net/wiki/Kyllocarus

Type species: Kyllocarus reticulatus Wang, Wei \& Yang, 2012, sp. n.

The new genus with flattened fusiform body, palp genual seta strongly angular prodorsal shield lacking scapular setae (sc) and tubercles, strong wide frontal lobe over gnathosoma, legs normal, except leg II lacking genual seta l'. Sternal apodeme present; opisthosoma differentiated into broader smooth dorsal annuli and narrower microtuberculate ventral annuli; empodium simple; genitalia very close to coxae, bearing two ranks of numerous ridges.

Diagnosis. This cecidophyine mite is very near to Kolacarus in that the genual seta $l$ " is absent on Leg II; however, it differs from other cecidophine mites in that palp genual seta $d$ is bent or crooked, possibly minutely bifurcate. It differs from Kolacarus in that the mite has a wide, strong frontal lobe projecting from the prodorsal shield over the gnathosoma; Kolacarus has a normal palp genual seta $d$, and no frontal lobe on the anterior prodorsal shield.

Etymology. Kyllo- from Gr. Kyllos, crooked + -carus from Acarus; the name is masculine.

\section{Kyllocarus reticulatus sp. $\mathbf{n}$.}

urn:Isid:zoobank.org:act:7358B76E-13F4-4968-82F4-2500479953D0

http://species-id.net/wiki/Kyllocarus_reticulatus

Figs 1-7

Diagnosis. Body fusiform, white translucency or yellow. Gnathosoma curved obliquely downward, dorsal genual setae (d) bend forming obtuse angle at middle. Prodorsal 


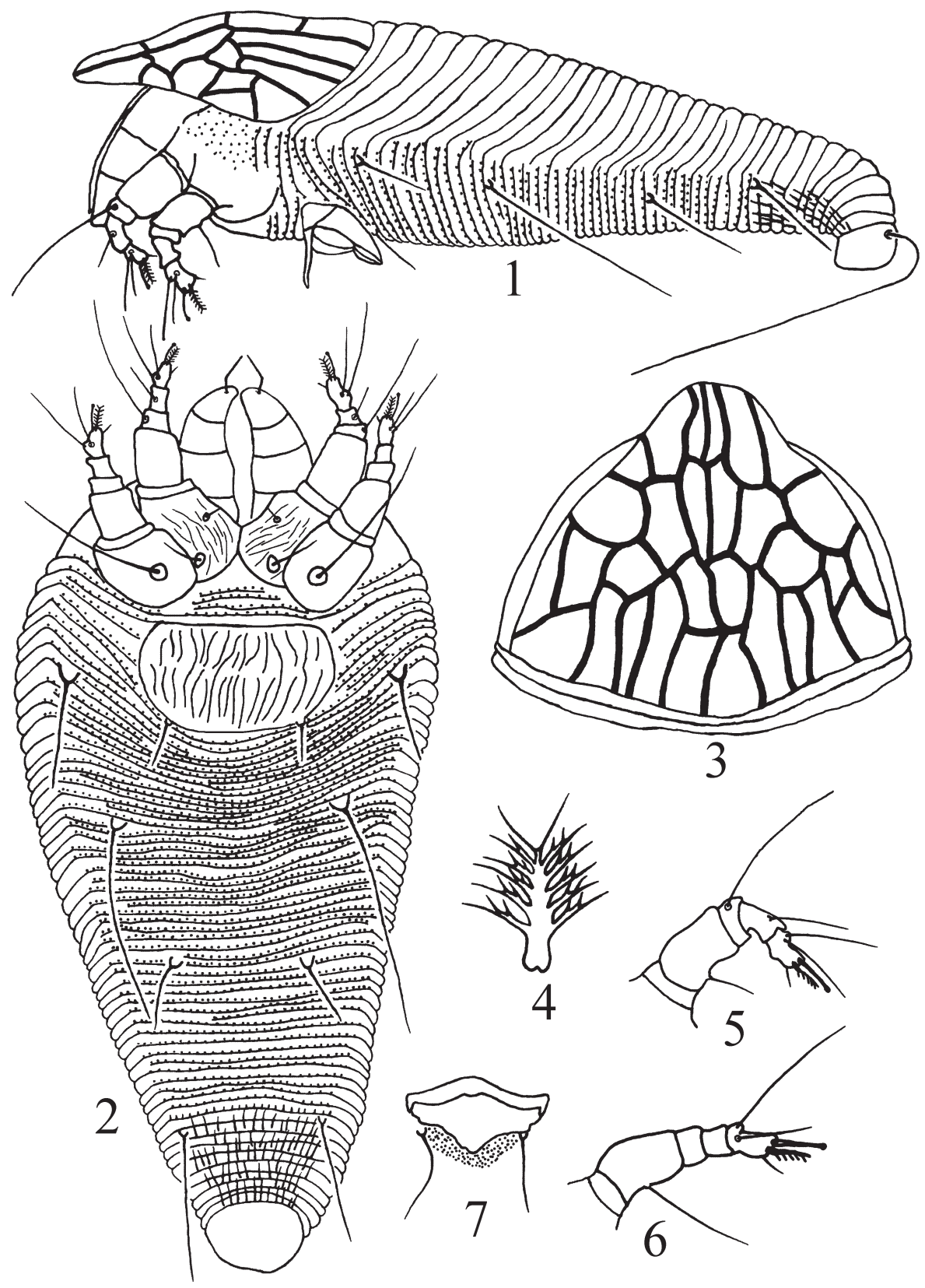

Figures I-7. Kolacarus reticulatus sp. n. I lateral view of female $\mathbf{2}$ ventral view of female $\mathbf{3}$ anterior dorsal view of female $\mathbf{4}$ tarsal empodium $\mathbf{5}$ leg I $\mathbf{6}$ leg II $\mathbf{7}$ male genitalia

shield with frontal lobe present; all lines bold and connected with transverse lines forming network; scapular tubercles and setae absent. Coxisternal plates sculptured with lines, prosternal apodeme present, coxigenital annuli 4. Legs segments normal, 
legs II with genual setae ( $\left.l^{\prime}\right)$ absent, tarsal empodium entire, 6-rayed, tarsal solenidion knobbed. Dorsal opisthosoma with shallow median furrow, dorsal annuli smooth; ventral annuli with rounded microtubercles, setae $h 1$ absent. Female genitalia coverflap with two rows of ridges

Description. Female $(\mathrm{n}=11)$. Body fusiform, white translucency or yellow, 172 (150-204), 75 (69-79) wide, 60 (54-63) thick.

Gnathosoma. Curved obliquely downward, 30 (28-31), coxal setae (ep) 6 (6-7), dorsal genual setae $(d)$ bend forming obtuse angle at middle, $11(10-12)$; cheliceral stylets $31(30-33)$.

Prodorsal shield. 63 (58-70), 69 (65-74) wide, frontal lobe present; all lines bold; median, admedian and submedian lines complete, connected with transverse lines forming network; scapular tubercles and setae absent.

Coxisternal plates. Prosternal apodeme present, coxisternal plates I and II sculptured with lines; anterolateral setae on coxisternum I (1b) 3 (3-4), 13 (12-13) apart; proximal setae on coxisternum I (1a) 5 (5-6), 13 (12-13) apart; proximal setae on coxisternum II (2a) 31 (29-33), 29 (29-30) apart. Coxigenital annuli 4.

Legs. Segments normal. Legs I 34 (30-37), trochanter 2 (2), femur 11 (10-11), femoral setae (bv) 13 (10-15); genu 5 (4-5), genual setae (l') 30 (29-32); tibia 7 (6-8), tibial setae $(l)$ located laterally and distally, 15 (13-18); tarsus 8 (7-9), inner fastigial tarsal setae $\left(f t^{\prime}\right) 27$ (25-28), outer fastigial tarsal setae $\left(f t^{\prime}\right) 18$ (15-20), unguinal tarsal setae $(u) 5$ (4-5); tarsal empodium entire, 12 (11-13), 6-rayed, tarsal solenidion 7 (6-8), knobbed. Legs II 27 (26-30), trochanter 2 (2), femur 10 (10-11), femoral setae (bv) 23 (20-25); genu 4 (4-5), genual setae (l') absent; tibia 4 (4-5); tarsus $7(6-7)$, inner fastigial tarsal setae ( $f t) 24(23-25)$, outer fastigial tarsal setae $\left(f t^{\prime}\right) 14$ (13-15), unguinal tarsal setae $(u) 4(4-5)$; tarsal empodium entire, 6 (5-7), 6-rayed, tarsal solenidion 7 (7-8), knobbed.

Opisthosoma. Dorsum with shallow median furrow, dorsal annuli 43, smooth; ventral annuli 63, with rounded microtubercles; setae $c 223$ (20-25), on ventral annulus 10th; setae $d 71$ (63-79), 42 (41-43) apart, on ventral annulus 22th; setae $e 11$ (8-13), 24 (23-25) apart, on ventral annulus 38th; setae $f 24$ (22-25), 24 (24-25) apart, on 10th ventral annulus from rear; setae $h 1$ absent, setae $h 231$ (26-39).

Female genitalia. Near coxisternal plates, coverflap with two rows of ridges, 24 (2325), 43 (38-49) wide, proximal setae on coxisternum III (3a) 9 (9-10), 23 (23-24) apart.

Male $(\mathrm{n}=2)$. Body fusiform, 120-140, 58-61 wide.

Prodorsal shield. 53-55, 55-57 wide, frontal lobe present; all lines bold; median, admedian lines and submedian lines complete, connected with transverse lines forming network; scapular tubercles and setae absent.

Coxisternal plates. Prosternal apodeme present, coxisternal plates I and II sculptured with lines; anterolateral setae on coxisternum I (1b) 3, 11 apart; proximal setae on coxisternum I (1a) 5, 11 apart; proximal setae on coxisternum II (2a) 27, 28 apart. Coxigenital annuli 4.

Legs. Segments normal. Legs I 30, trochanter 2, femur 10, femoral setae (bv) 12; genu 4, genual setae $\left(l^{\prime}\right) 27$; tibia 6 , tibial setae $(l)$ located laterally and distally, 12; 
tarsus 7 , inner fastigial tarsal setae $\left(f t^{\prime}\right) 24$, outer fastigial tarsal setae $\left(f t^{\prime}\right) 15$, unguinal tarsal setae $(u)$ 4; tarsal empodium entire, 10, 6-rayed, tarsal solenidion 6, knobbed. Legs II 26, trochanter 2, femur 10, femoral setae $(b v)$ 18; genu 4, genual setae (l') absent; tibia 4; tarsus 6 , iner fastigial tarsal setae $\left(f t^{\prime}\right) 21$, outer fastigial tarsal setae $\left(f t^{\prime}\right)$ 12, unguinal tarsal setae $(u)$ 4; tarsal empodium entire, 5, 6-rayed, tarsal solenidion 7, knobbed.

Opisthosoma. Dorsum with shallow median furrow, dorsal annuli 42, smooth; ventral annuli 62, with rounded microtubercles; setae $c 220$, on ventral annulus 10th; setae $d$ 57, 40 apart, on ventral annulus 22th; setae $e$ 7, 21 apart, on ventral annulus 38th; setae $f 20,21$ apart, on 10th ventral annulus from rear; setae h1 absent, setae $h 227$.

Male genitalia. Near coxisternal plates, 36 wide, proximal setae on coxisternum III (3a) 8, 23 apart.

Type material. Holotype female, China: Zhejiang, Longquan City, Fengyangshan National Nature Reserve (27 $\left.53^{\prime} \mathrm{N}, 119^{\circ} 11^{\prime} \mathrm{E}\right), 27$. VII. 2007, collected by Guo-Quan Wang, from Lithocarpus brevicaudatus (Skan) Hayata (Fagaceae). Paratypes, 10 females and 2 males.

Distribution. China (Zhejiang).

Etymology. The species is named after the network-form of the prodorsal shield.

\section{Tribe Colomerini Newkirk \& Keifer, 1975 \\ Genus Gammaphytoptus Keifer, 1939}

Type species: Gammaphytoptus camphorae Keifer, 1939.

\section{Gammaphytoptus schimae sp. n.}

urn:lsid:zoobank.org:act:CEC41DF7-A6BB-4871-9D06-FABC3DDAC2 19

http://species-id.net/wiki/Gammaphytoptus_schimae

Figs $8-13$

Diagnosis. Body fusiform, yellow. Gnathosoma curved obliquely downward, dorsal genual setae $(d)$ bend forming obtuse angle at middle. Prodorsal shield with frontal lobe present; all lines bold and connected with transverse lines forming network; scapular tubercles and setae absent. Coxisternal plates sculptured with lines, prosternal apodeme present, coxigenital annuli 4. Legs segments normal, legs II with genual setae (l') absent, tarsal empodium entire, 6-rayed, tarsal solenidion knobbed. Dorsal opisthosoma with shallow median furrow, dorsal annuli smooth; ventral annuli with rounded microtubercles, setae $h 1$ absent. Female genitalia coverflap with two rows of ridges.

Description. Female ( $\mathrm{n}=11)$. Body fusiform, yellow, 183 (169-200), 71 (65-78) wide, 44 (38-52) thick.

Gnathosoma. Curved obliquely downward, 34 (28-35), coxal setae (ep) 2 (2-3), dorsal genual setae (d) 10 (9-11); cheliceral stylets 30 (28-32). 


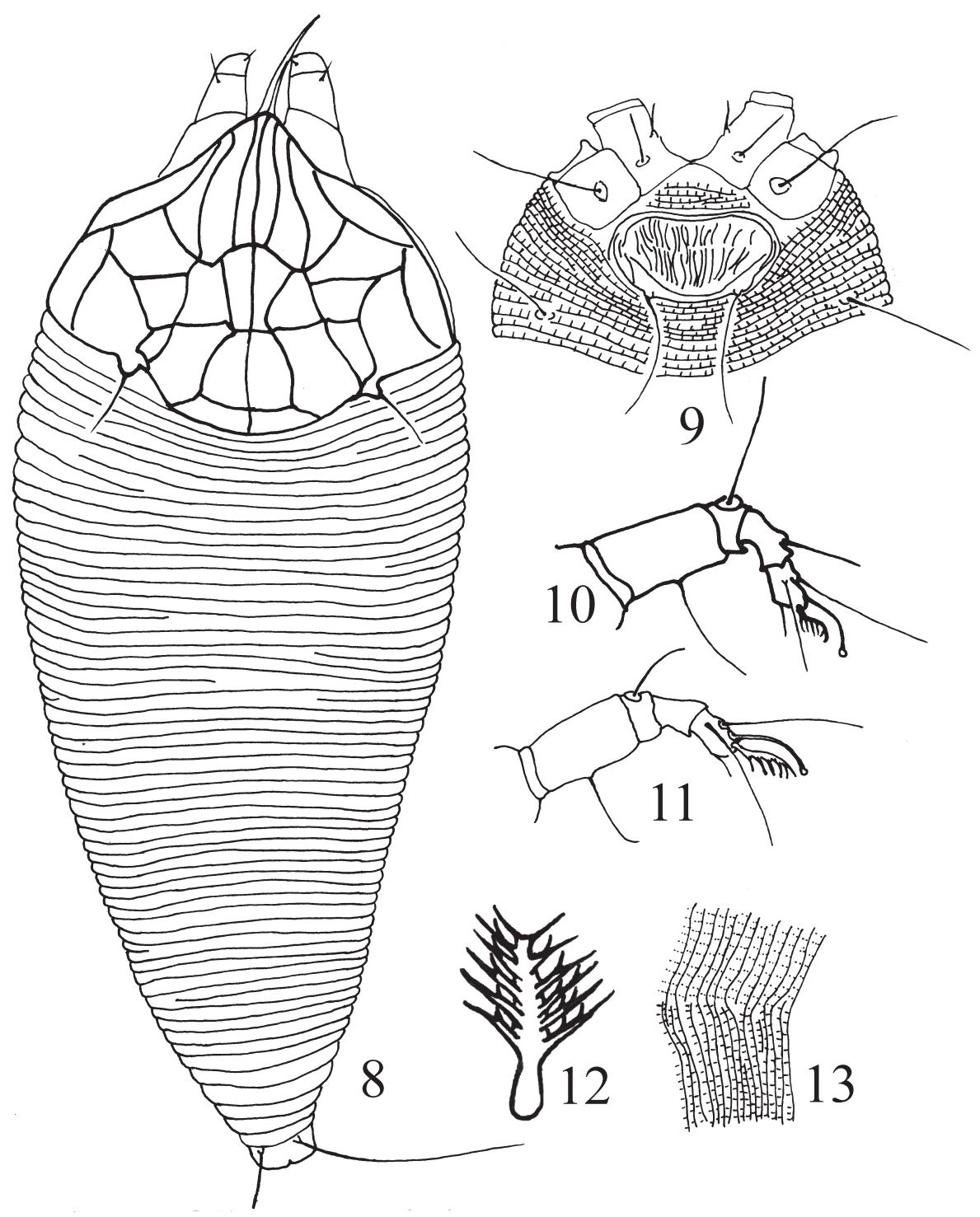

Figures 8-13. Gammaphytoptus schimae sp. n. 8 dorsal view of female $\mathbf{9}$ coxigenital area of female $\mathbf{1 0}$ leg I I I leg II I 2 tarsal empodium I 3 lateral view of annuli.

Prodorsal shield. 51 (48-52), 55 (50-63) wide, frontal lobe present; median, admedian and submedian lines complete, connected with three transverse lines forming network; scapular tubercles placed at rear shield margin, 35 (31-39) apart, scapular setae (sc) 8 (8-9), directed backward and divergence.

Coxisternal plates. Prosternal apodeme present, coxisternal plates smooth; anterolateral setae on coxisternum I (1b) 8 (7-9), 13 (12-13) apart; proximal setae on coxis- 
ternum I (1a) 25 (19-31), 15 (14-15) apart; proximal setae on coxisternum II (2a) 35 (28-39), 28 (27-30) apart. Coxigenital annuli 4.

Legs. Segments normal. Legs I 36 (34-38), trochanter 2 (2), femur 12 (12-13), femoral setae (bv) 18 (15-22); genu 4 (4-5), genual setae (l’) 35 (31-40); tibia 10 (9-10), tibial setae $(l)$ located $1 / 4$ from apical, $8(7-8)$; tarsus $8(7-8)$, iner fastigial tarsal setae (ft) 20 (18-23), outer fastigial tarsal setae $\left(f t^{\prime}\right) 25$ (23-28), unguinal tarsal setae $(u) 5$ (56); tarsal empodium entire, 7 (7-8), 6-rayed, tarsal solenidion 10 (9-10), knobbed. Legs II 31 (29-34), trochanter 2 (2), femur 11 (11-12), femoral setae (bv) 23 (19-27); genu 3 (3-4), genual setae (l') 10 (7-12); tibia 7 (7-8); tarsus 8 (7-8), inner fastigial tarsal setae $\left(f t^{\prime}\right) 10(9-12)$, outer fastigial tarsal setae $\left(f t^{\prime}\right) 25$ (22-29), unguinal tarsal setae $(u)$ 5 (5-6); tarsal empodium entire, 8 (8-9), 6-rayed, tarsal solenidion 10 (9-11), knobbed.

Opisthosoma. Dorsum evenly rounded, dorsal annuli 59-60, with semi-translucency elongated microtubercles; ventral annuli 81, with filament microtubercles; setae c2 38 (35-40), on ventral annulus 13th; setae $d 45$ (37-50), 43 (38-45) apart, on ventral annulus 28th; setae $e 27$ (23-32), 25 (23-26) apart, on ventral annulus 44th; setae $f 38$ (34-45), 23 (21-26) apart, on 7 th ventral annulus from rear; setae $h 1$ absent, setae h2 57 (53-65).

Female genitalia. Near coxisternal plates, coverflap with two rows of ridges, 17 (16-18), 30 (29-22) wide, proximal setae on coxisternum III (3a) 20 (17-25), 13 (13-14) apart.

Male. Unknown.

Type material. Holotype female, China: Zhejiang, Longquan City, Fengyangshan National Nature Reserve (27 $53^{\prime}$ N, $119^{\circ} 11^{\prime}$ E), 28. VII. 2007, collected by Guo-Quan Wang, from Schima superba Gardn. et Champ. (Theaceae). Paratypes, 8 females.

Distribution. China (Zhejiang).

Etymology. The species is named from the generic name of the type host plant.

Remarks. This new species is similar to G. zuihoensus Huang \& Wang, 2004, but they can be easily separated as follows: in $G$. schimae, median line is complete, setae $h 1$ is absent and infesting Schima superba Gardn. \& Champ.; in G. zuihoensus, median line is incomplete, setae $h 1$ is present and infesting Machilus zuihoensis Hay. var. zuihoensis (Huang and Wang 2004).

\section{Acknowledgements}

The authors sincerely thank Professor James W. Amrine JR. and the anonymous reviewers for their valuable remarks. Undoubtedly, Prof. Amrine's very valuable suggestions for the authers to erect the new genus. We would like to thank Prof. Hua $\mathrm{Li}$ (College of Agriculture, Guangxi University) for identifying the host plants. This work was supported by the National Natural Science Foundation of China (Grant No. 31160431), the Key Project of Chinese Ministry of Education (Grant No. 211134) and Major Program of Ministry of Science and Technology of the People's Republic of China (Grant No. 2006FY110500-2). 


\section{References}

Amrine JWJr, Stasny TA, Flechtmann CHW (2003) Revised keys to world genera of Eriophyoidea (Acari: Prostigmata). Indira Publishing House, Michigan, U.S.A., 244 pp.

Boczek J, Chandrapatya (1998) A studies on eriophyoid mites (Acari: Eriophyoidea). XXII. Acarologia 39(2): 135-142.

de Lillo E, Craemer C, Amrine JW Jr, Nuzzaci G (2010) Recommended procedures and techniques for morphological studies of Eriophyoidea (Acari: Prostigmata). Experimental and Applied Acarology 51: 283-307. doi: 10.1007/s10493-009-9311-x

Huang KW (2001) Eriophyoid mites of Taiwan: description of eighty-six species from the Tengchih area. Bulletin of the National Museum of Natural Science 14: 1-84.

Huang KW, Cheng LS (2005) Eriophyoid mites of Hainan, China (Acari: Eriophyoidea). Formosan Entomology 25: 269-301.

Huang KW, Wang CF (2004) Eriophyoid mites of Taiwan: Description of nine species of Cecidophyinae and Eriophyinae from Hueysuen (Acari: Eriophyoidea). Plant Protection Bulletin 46: 55-68.

Huang KW, Wang CF (2009) Eriophyoid mites (Acari: Eriophyoidea) of Taiwan: thirty-seven species from Yangmingshan, including one new genus and twenty-two new species. Zootaxa 1986: 1-50.

Keifer HH (1939) Eriophyid Studies VI. Bulletin of the California Department of Agriculture 28: 418-419, 423.

Keifer HH (1944) Eriophyid Studies XIV. Bulletin of the California Department of Agriculture 33: 26-27, 37.

Keifer HH (1959a) New eriophyid mites. Annals of Entomological Society of America 52: 649-650.

Keifer HH (1959b) Eriophyid Studies XXVIII, Occasional Paper 2. California Department of Agriculture 8-9.

Keifer HH (1962) Eriophyid Studies B-5. Bureau Entomology, California Department of Agriculture $2-3$.

Keifer HH (1966) Eriophyid Studies B-20. Bureau Entomology, California Department of Agriculture 17-20.

Kuang HY (1986) Agricultural Acarology. Agricultural Publishing House, Beijing, China, 290 pp.

Kuang HY (1987) Five new species of Eriophyidae from China. Journal of Nanjing Agricultural University 9(2): 36-41.

Kuang HY (1995) Economic insect fauna of China. Acari: Eriophyoidea (1). Fasc. 44. Science Press, Beijing, 198pp.

Kuang HY (1997) Four new species of Eriophyidae (Acari: Eriophyoidea) from China. Entomotaxonomia 19(1): 74-78.

Kuang HY (1998) A new genus and four new species of the subfamily Phyllocoptinae (Acari: Eriophyoidea) from the People Republic of China. Acarologia 39(1): 57-62.

Kuang HY, Hong XY, Luo WD (1992) Two new species of the subfamily Cecidophyinae from China (Acari: Eriophyidae). Acta Zootaxonomica Sinica 17(1): 57-60.

Kuang HY, Luo GH, Wang AW (2005) Fauna of Eriophyid Mites from China (II). China Forestry Publishing House, Beijing, China, 176pp. 
Livshitz IZ, Mitrofanov VI, Vasil'yeva EAA (1979) Contribution to the fauna of quadripedal oak mites (Eriophyidae, Acariformes). Zoologicheskii Zhurnal 58: 344-349.

Nalepa A (1898) Neue Gallmilben (17. Fortsetzung). Anzeiger der kaiserlichen Akademie Wissenschaften. Mathematische-naturwissenschaftliche Klasse, Wien 35: 233-235.

Song ZW, Xue XF, Hong XY (2008) Eriophyoid mite fauna (Acari: Eriophyoidea) of Gansu Province, northwestern China with descriptions of twelve new species. Zootaxa 1756: 1-48.

Wang GQ, Wei SG, Yang D (2009) Six new eriophyoid mites (Acari: Eriophyoidea) associated with Ficus spp. (Moraceae) from China. Zootaxa 2201: 49-62.

Wei SG, Wang GQ, Li DW, Ou SS (2009) Eriophyoid Mites of Guangxi, China (Acari: Eriophoidea). Guangxi Science and Technique Press, Guangxi, China, 329pp

Xue XF, Wang Z, Song ZW, Hong XY (2009) Eriophyoid mites on Fagaceae with descriptions of seven new species and eleven new species (Acari: Eriophyoidea). Zootaxa 2253: 1-95. 